\title{
De uma sociedade desumanizada que temos para uma sociedade justa e solidária, com saúde ambiental, que precisamos
}

\author{
From our inhuman society to a fair and solidarity-based society with \\ healthy environment such as we need
}

\section{Desde una sociedad inhumana como la que tenemos a una sociedad justa y solidaria con salud ambiental como necesitamos}

Carlos José Saldanha Machado | carlos.saldanha@icict.fiocruz.br

Fundação Oswaldo Cruz (Fiocruz), Instituto de Comunicação, Informação Científica e Tecnológica em Saúde (Icict). Rio de Janeiro, Brasil.

Autor do projeto editorial Reciis (2006)

Vivemos um momento histórico de profundas mudanças, contradições e radicalismos ${ }^{1-4}$. Vistos de uma forma fragmentada, são inúmeros acontecimentos que se sobrepõem nessa dinâmica. Contudo, em uma perspectiva holística, podemos compreender tais acontecimentos como configurações interdependentes de uma grande causa: os limites paradigmáticos de um modelo de civilização e desenvolvimento que vem sendo consolidado no último século e intensificado nos últimos 50 anos, com a predominância do capitalismo neoliberal que tem se esforçado para uniformizar a humanidade através de uma plêiade de ações, em escala planetária, conhecida como globalização $0^{1,5-8}$. Esse termo refere-se àqueles processos que tendem a criar e consolidar uma economia mundial unificada - transformando recursos naturais em matérias-primas e energia, sem levar em consideração os sistemas biológicos e físicos que sustentam a vida na Terra. $\mathrm{O}$ aumento do fluxo de capitais torna-se mais importante do que as atividades de produção e de comercialização de produtos e a sua consolidação se dá através de uma complexa rede de comunicações ${ }^{9-10}$. Integrada por tecnologias de telecomunicações, espaciais (foguete espacial e satélite artificial) e de informação e comunicação (internet e World Wide Web, ou Web), essa rede abarca todo o globo e liga localidades distantes, mesmo não penetrando em todas as relações sociais dos territórios de cada parte de um planeta habitado por mais de sete bilhões de pessoas 5,9. Tais processos tendem a tornar o mundo mais interdependente, juridicamente regulado através de regimes os mais variados, que incluem formas de coordenação e de organização públicas e privadas; mas isso não significa que estamos nos movendo inexoravelmente em direção a um governo único mundial, a um único modelo de governança, nem significa o fim dos estados nacionais como atores importantes nas dinâmicas de enraizamentos territoriais da globalização ${ }^{1,7,9}$. 
Os governos nacionais, assessorados por economistas que se apresentam como mestres da arte de antecipar tendências através da manipulação de equações baseadas em suposições irrealistas ${ }^{12}$, fazem escolhas monetárias de políticas macroeconômicas, reforçam um sistema tributário que amplia a acumulação capitalista de poucos ao invés de promover a distribuição de renda para muitos, liberam os mercados financeiros, viabilizando a financeirização da economia, privatizam as empresas nacionais, desregulam a regulamentação comercial, flexibilizam os mercados de trabalho, reduzem os orçamentos da proteção social, convergem para a criação de novos mercados como o da bioeconomia, e mantêm, ao mesmo tempo, uma forma usual e consensual, ainda que genérica, de apostar no ‘desenvolvimento’ como uma ideia correlata de crescimento, de progresso e de evolução humana, o ideário de todos os processos bem-sucedidos. Tal ideia, que postula a infinitude dos recursos naturais e a infinitude do desenvolvimento rumo ao futuro, constitui um determinado tipo de ser humano, de profissional, de relação com a natureza e um modelo de sociedade assentado na cultura da dilapidação do capital natural como um dos valores da economia de mercado'.

Com o passar dos anos, as evidências da exaustão da dimensão econômica do modelo de globalização neoliberal, sobretudo em função das sucessivas crises financeiras inerentes ao capitalismo, fazem-se notar em sua incapacidade de gerar riqueza sem gerar, concomitantemente, e de forma crescente, exclusão e desigualdade, suicídio, tentativa de suicídio e aumento de vários tipos de doença mental, crimes, pauperização, violência, degradação ambiental, trabalho escravo, insegurança alimentar, poluição, perda de biodiversidade, aumento do tráfico de animais e do espraiamento de espécies exóticas invasoras. O aumento do consumo de combustíveis fósseis (petróleo, gás natural e carvão mineral); a contaminação dos solos por hidrocarbonetos, a emissão de gases de efeito estufa (dióxido de carbono [CO2], metano [CH4], óxido nitroso [N2O], perfluorocarbonos [PFCs], clorofluorcarbono [CFC], hidrofluorcarbonetos [HFCs], hexafluoreto de enxofre [SF6]) provocam, entre outros problemas, mudanças climáticas e aumento da temperatura do planeta. Além disso, observam-se conflitos socioambientais, emergência de novas doenças e epidemias, e reemergência de outras que haviam desaparecido, crises humanitárias que não param de se multiplicar, racismo institucional, expansão do mercado de planos de saúde com cobertura restrita e judicialização da saúde, expansão do setor financeiro dos seguros, emergência do fenômeno religioso de crenças milenaristas e apocalípticas, a proliferação de igrejas evangélicas e a expansão da corrupção na democracia ou a dilapidação do patrimônio público ${ }^{3,5}$,11-19.

Diante dessa realidade transnacionalizada, as ciências são cada vez mais convocadas e cobradas, no espaço público, a resolver esse ou aquele problema de sociedade, e aqueles profissionais que comparecem tornam-se atores indispensáveis na busca de formas exequíveis de se alcançar uma sociedade sustentável-2, 9, 21-33. As ciências apoiam-se em equipes, infraestrutura e laboratórios cada vez mais exigentes em termos de capital para a realização das pesquisas, de forma semelhante às que são desenvolvidas nos laboratórios industriais, em arranjos institucionais macroepistêmicos ${ }^{34-35}$ com atores que se voltam tanto para a troca e o processamento de informações, por meio de rede de conexão global, a internet, como para a validação do conhecimento resultante de atividades de levantamento de amostras, monitoramento, observação e análise das transformações dos complexos de sistemas que sustentam a vida na Terra. São pesquisas sofisticadas e intensivas em capital, majoritariamente realizadas com recursos de agências de fomento internacionais e nacionais, grande parte coordenada na forma de redes colaborativas ${ }^{36}$, que ampliam e aprimoram a qualidade das evidências para reforçar a constatação de que esses sistemas estão em acelerado declínio e, em muitos aspectos, de forma irreversível.

De fato, uma das responsabilidades centrais de homens e mulheres de ciências, sobretudo em uma sociedade tão desigual como a brasileira ${ }^{37-39}$, que atravessa um momento político de golpe de Estado parlamentar midiático jurídico neoliberal ${ }^{2}$, é contribuir para a passagem de uma sociedade desumanizada que temos para uma sociedade democrática, justa e solidária, com saúde ambiental que precisamos. A cidadania de cada um exige a vivência e a responsabilidade diária pelos rumos da política, do governo, 
da saúde, da educação e do meio ambiente porque qualquer que seja o desenvolvimento das dependências transnacionais, a vida material de cada um está ligada, antes de mais nada, à organização e ao funcionamento de uma economia, ligados, por sua vez, às ações ou à inércia do poder estatal7 ${ }^{7}$ É preciso agir localmente, de forma eficiente e eficaz, para superar a crescente degradação do planeta, com a adoção da sustentabilidade como modelo de justiça social, equidade intergeracional e equilíbrio ambiental ${ }^{1,11}$.

Mas é preciso ter em mente que as ciências são atividades humanas, práticas envolvidas em teias socioculturais, não tendo respostas definitivas para todas as questões e problemas enfrentados; uma de suas características é a possibilidade de serem questionadas por não produzirem verdades indiscutíveis e de se transformarem em função de processos históricos, políticos, econômicos, sociais e de ambientes institucionais favoráveis, ou não, ao seu desenvolvimento ${ }^{42}$. Admitir o contrário dessas características socioantropológicas seria comungar da ideologia cientificista construída na Europa do século XIX, que outorgou à ciência um caráter neutro e universal, transformando-a em uma forma de altruísmo. Acreditavase ser desprovida de valores sociais e de interesses econômicos, com homens e mulheres empreendendo ações benéficas de alcance universal ${ }^{39} \mathrm{em}$ pleno processo de expansão colonial ou "missão civilizatória".

Em nossa era do paroxismo das assimetrias, um periódico científico interdisciplinar como a Reciis precisa participar de coalizões e pactos que veem sendo construídos para um novo estilo de desenvolvimento e se constituir como fonte atualizada para o início de novas pesquisas e legitimação de novos campos de estudos, ofertando a seus leitores um conjunto de problemas, objetos, abordagens e reflexões críticas ancoradas, empiricamente, na realidade do vasto território nacional, socioambientalmente marcado pelo septuagenário modelo desenvolvimentista que ignora, ou desvaloriza, os custos e benefícios ambientais, mas sempre privatizam os lucros e socializam os efeitos da dilapidação do capital natural ${ }^{1,28}$. Não obstante a natureza pública de uso comum dos recursos naturais como o ar atmosférico, a flora, o solo, o subsolo, a fauna, as águas interiores, superficiais e subterrâneas, os mares e os oceanos..., a sua crescente degradação é resultado de danos e riscos relacionados à construção e ao uso de novas técnicas, tecnologias, processos e métodos de produção $0^{19-20,40-43}$ que, por sua vez, utilizam substâncias, matéria e energia em um ritmo incompatível com a resiliência do planeta, ou seja, a capacidade de resposta e de recuperação de seus sistemas às perturbações sofridas, de aprender, se auto-organizar e alcançar múltiplos equilíbrios. Eis uma forma da Reciis atrair e ampliar a atenção de diferentes grupos sociais de pesquisadores/autores, ao situar os resultados das pesquisas publicadas no seu grau de originalidade em relação ao conhecimento já acumulado em sua extensa área de atuação. Trata-se de um conhecimento construído a partir de uma infraestrutura com configurações que vão do infinitamente pequeno, como nanopartículas em reagentes químicos, ao imensamente grande, como navios, aviões e satélites, passando por bancos de dados com séries estatísticas, algoritmos de softwares que alimentam o funcionamento de potentes computadores na sistematização e na análise de volumosos dados diluvianos ${ }^{1}$.

Movimentando-se nessa direção, a Reciis caminhará a passos largos para a atualização do papel que é seu desde a sua concepção original: constituir um dos principais veículos formais de comunicação científica ao reunir parte expressiva da produção mais representativa da sua heterogênea área de conhecimento e dar visibilidade e prestígio aos pesquisadores entre um público altamente especializado. Para tanto, o aprimoramento e/ou criação de critérios de qualidade da Reciis é um processo contínuo deste espaço online que participa de um mundo editorial extremamente competitivo de acesso livre à informação científica, mundo que gravita em torno do conhecimento como patrimônio comum da espécie humana e não como objeto de apropriação privada e patenteada.

Uma revista científica interdisciplinar da centenária Fundação Oswaldo Cruz - enraizada no território nacional e avessa aos valores do neoliberalismo porque incompatíveis com o pacto social constitucionalmente firmado ${ }^{41}$ após um longo período de ditadura militar (1964-1985) - deve desenvolver meios de trabalhar de maneira integrada e intersetorial as questões associadas ao desenvolvimento pautado nos conceitos de sustentabilidade, transparência na política e integridade na vida pública e no serviço público1 para se 
engajar na luta política de libertação das coletividades humanas da fome, da pobreza extrema e de doenças que podem ser prevenidas e combatidas com um Sistema Único de Saúde (SUS) que garanta a assistência integral e totalmente gratuita para a totalidade da população, e estrategicamente com planejamento. Esse engajamento - um imperativo face ao recrudescimento do conservadorismo, da violência e do populismo, que rejeita o pluralismo, nas Américas e na Europa ${ }^{3-4,47}$ - se concretiza dentro dos limites da ética, da moral e da lei, ao viabilizar o exercício de um dos papéis das ciências em um Estado democrático de direito: produzir informações e conhecimentos que permitam aos atores da dinâmica territorial uma melhor tomada de decisão nos processos de formulação, implementação, avaliação ou, simplesmente, recusa de políticas públicas que afetam a vida em sociedade. Mas, no Brasil, o Estado democrático de direito não está vigorando desde o dia 12 de maio de 2016, quando o Senado confirmou a admissibilidade do processo de impeachment da presidenta da República (eleita com 54.501.118 de votos dos mais de 110 milhões de eleitores) que havia sido votada pela Câmara dos Deputados no dia 17 de abril de 2016, em um espetáculo patético comentado pelas mais importantes mídias internacionais. Entre elas, CNSNews, CNN, The New York Times, The Times, The Economist, The Guardian, El País, Volkskrant, Die Zeit, Der Spiegel, L’Express, e Libération se surpreenderam com a mediocridade e o fundamentalismo dos deputados.

Como um organismo vivo, um periódico científico deve saber se adaptar, para sobreviver, às mudanças do ambiente na era do paroxismo das assimetrias, a era em que os humanos assumiram o papel de força motriz das alterações globais do planeta, a ponto de rivalizar com as grandes forças da natureza, o antropoceno $0^{1,1,28}$. Mas, nesse processo adaptativo, é preciso ter claro que tanto as ideologias liberais quanto as socialistas não souberam lidar com, e nem mesmo contemplaram no seu projeto político, a degradação ambiental em processos industriais, com a geração de fumaça, resíduos sólidos e efluentes líquidos no solo e nos corpos hídricos; muito pelo contrário, ambos, o capitalismo industrialista e o coletivismo industrialista colocaram em operação um modelo industrial agressivo aos valores ambientais de vida em sociedade. É preciso agir rapidamente para evitar mais catástrofes ambientais que já veem acontecendo, de forma exponencial, desde meados do século XX.

Em síntese, é imperativo que sejamos responsáveis moralmente e legalmente pelo mundo em que vivemos, ou seja: pela Terra em que os Homens se movem e da qual extraem a matéria-prima com que fabricam coisas; pelas barreiras artificiais que os Homens interpõem entre si e entre eles e a própria natureza; pelo conjunto de artefatos e de instituições criadas que permitem que eles estejam relacionados entre si sem que deixem de estar simultaneamente separados; pelo espaço institucional democrático que deve sobreviver ao ciclo natural da natalidade e mortalidade das gerações.

\section{Referências}

1. Machado CJS. Desenvolvimento sustentável para o antropoceno: um olhar panorâmico. Rio de Janeiro: E-Papers; 2014.

2. Machado CJS, Vilani RM, organizadores. Temas e problemas da vida em sociedade no brasil. Rio de Janeiro: E-Papers; 2016.

3. Mishra P. Age of anger: a history of the present. New York: Farrar, Straus and Giroux; 2017.

4. Scheidel W. The great leveler: violence and the history of inequality from the stone age to the twentyfirst century. Princeton: Princeton University Press; 2017.

5. Baker D. Rigged: how globalization and the rules of the modern economy were structured to make the rich richer. Washington, DC: Center for Economic and Policy Research; 2016.

6. Harvey D. A brief history of neoliberalism. Nova York: Oxford; 2005.

7. Machado CJS, organizador. Ciências, políticas públicas e sociedade sustentável. Rio de Janeiro: E-Papers; 2012.

8. Vitali S, Glattfelder JB, Battiston S. The network of global corporate control. Plos One. 2011;6(10):1-6. 
9. Machado CJS, Vilani, RM. Governança climática no antropoceno: da rudeza dos fatos à esperança no Brasil. Rio de Janeiro: E-Papers; 2015.

10. Earle J, Moral C, Ward-Perkins Z. The econocracy: the perils of leaving economics to the experts. Manchester: Manchester University Press; 2016.

11. Block F, Somers MR. The power of market fundamentalism: Karls Polanyi's critique. Cambridge: Harvard University Press; 2014.

12. Davis W. The limits of neoliberalism: authority, sovereignty and the logic of competition. New York: Sage; 2014.

13. Merson MH, Black RE, Mills AJ, editors. Global health. diseases, programs, systems, and policies. Londres: Jones \& Bartlett Learning; 2012.

14. Ostry D, Loungani P, Furceri D. Neoliberalism: oversold? IMF Financ Dev. 2016;53(2):38-41.

15. Piketty T. Le capital au XXIe siècle. Paris: Seuil; 2013.

16. Sobreira RFF, Machado CJS, Vilani RM. A criminalização das religiões afro-brasileiras. Direitos Culturais. 2016;11(23):143-64.

17. World Bank. The cost of air pollution: strengthening the economic case for action. Washington, DC: World Bank: Institute for Health Metrics and Evaluation; 2016.

18. World Health Organization. Ambient air pollution: a global assessment of exposure and burden of disease. Genebra: WHO; 2016.

19. World Wildlife Fund. Living planet report 2016. New York: WWF; 2016.

20. Intergovernamental Panel on Climate Change. Climate change: first assessment report 1990. Cambridge, UK: Cambridge University Press; 1990.

21. Intergovernamental Panel on Climate Change. Climate change. Second assessment report 1995. Cambridge, UK: Cambridge University Press; 1995.

22. Intergovernamental Panel on Climate Change. Climate change. Third assessment report 2001. Cambridge, UK: Cambridge University Press; 2001.

23. Intergovernamental Panel on Climate Change. Climate change. Fourth assessment report 2007. Cambridge, UK: Cambridge University Press; 2007.

24. Intergovernamental Panel on Climate Change. Climate Change 2013. The Physical Science Basis. Summary for Policymakers. Genebra: WHO/IPCC; 2013.

25. Intergovernamental Panel on Climate Change. Climate Change 2014. Mitigation of Climate Change. Genebra: WHO/IPCC; 2014.

26. Issberner LR, Lena $\mathrm{P}$, editors. Brazil in the anthropocene: conflicts between predatory development and environmental policies. Londres: Routledge; 2016.

27. Machado CJS Contribuições de um cientista social para aproximar a virologia ambiental das políticas públicas no Brasil. In: Kerbauy MTM; Andrade THN; Hayashi CRM, organizadores. Ciência, Tecnologia e Sociedade no Brasil. Campinas: Alínea; 2012, p. 221-249.

28. Machado CJS, Ferreira CA. Levantamento de áreas extrativistas das microbacias dos rios Cautário e São Miguel, Vale do Guaporé, Rondônia, para a criação de reservas extrativistas. Relatório Final de Pesquisa IEF/IBDF-MMA/Banco Mundial. Porto Velho: Instituto Estadual de Florestas de Rôndonia; 1987.

29. Sen A. The idea of justice. Cambridge, UK: Belknap Harvard; 2009.

30. Sen A. Desenvolvimento como liberdade. São Paulo: Companhia das Letras; 2010.

31. Shiva V. Seed sovereignty, food security: women in the vanguard of the fight against GMOs and corporate agriculture. Berkeley, CA: North Atlantic Books; 2016.

32. Shiva V. Who really feeds the world? The failures of agribusiness and the promise of agroecology. Berkeley, CA: North Atlantic Books; 2016.

33. Zélem MC, Beslay C. eds. La sociologie de l'énergie: gouvernance et pratiques sociales. Paris: CNRS Éditions; 2015. 
34. Cetina KK. Culture in global knowledge societies: knowledge, cultures and epistemic cultures. Interdiscipl Sci Rev. 2007;32(4):361-75.

35. Olson GM, Zimmerman A, Bos N, editores. Scientific collaboration on the internet. Cambridge, EUA: The MIT Press; 2008.

36. Shrum W, Genuth J, Chompalov I. Structures of scientific collaboration. Cambridge, EUA: The MIT Press; 2007.

37. Arretche M. Trajetórias da desigualdade: como o Brasil mudou nos últimos 50 anos. São Paulo: Unesp; 2015.

38. Gobetti W, Orair RO. Tributação e distribuição da renda no Brasil: novas evidências a partir das declaraçõ̃es tributárias das pessoas físicas. Working paper n. 136. Brasília: Centro Internacional de Políticas para o Crescimento Inclusivo/Programa das Nações Unidas para o Desenvolvimento; 2016.

39. Machado CJS. Olhares acadêmicos sobre a invenção e a descoberta nas ciências. Rio de Janeiro: E-Papers; 2010.

40. Hughes TP. Human-Built World: how to think about technology and culture. Chicago: The University of Chicago Press; 2004.

41. Ikde D. Technology and lifeworld, from garden to earth. Bloomington: Indiana University Press; 1990.

42. Nye DE. Technology Matters. Questions to live with. Cambridge: The MIT Press; 2007.

43. Brasil. Casa Civil. Constituição da República Federativa do Brasil de 1988. Brasília, DF: Centro Gráfico; 1988. 